Supporting Information belonging to the manuscript:

\title{
On the Structure and the Spin States of Fe(III)- EDDHA Complexes
}

Mar Gómez-Gallego, ${ }^{*}{ }^{\perp}$ Israel Fernández, ${ }^{\perp}$ Daniel Pellico, ${ }^{\perp}$ Ángel Gutierrez ${ }^{\varnothing}$, Miguel A. Sierra, ${ }^{*, \perp}$

Departamentos de Química Orgánica y Química Inorgánica. Facultad de Química.

Universidad Complutense. 28040-Madrid, Spain.

margg@quim.ucm.es; sierraor@quim.ucm.es

Juan J. Lucena

Sección de Química Agrícola. Facultad de Ciencias, CVII. Universidad Autónoma, 28049-

Madrid, Spain

\footnotetext{
${ }^{\perp}$ Departamento de Química Orgánica

${ }^{\varnothing}$ Departamento de Química Inorgánica
} 
Cartesian coordinates (in $\AA$ ) and total energies (in a. u., non corrected zero-point vibrational energies included) of all the stationary points discussed in the text. All calculations have been performed at the B3LYP/6-31G*\&LANL2DZ//B3LYP/3$21 G^{*} \& L a n L 2 D Z$ level of theory.

2a: $E=-1379.68466$

\begin{tabular}{|c|c|c|c|}
\hline C & 3.268520 & ๑. 165051 & -0.060424 \\
\hline C & 4.664095 & 0.100351 & -0.079997 \\
\hline C & 5.440195 & 1.185552 & -0.482266 \\
\hline C & 4.790720 & 2.365400 & -0.874017 \\
\hline C & 3.407753 & 2.450905 & -0.859441 \\
\hline C & -4.664118 & 0.100381 & $\odot .079927$ \\
\hline C & -3.268544 & 0.165074 & ๑.060291 \\
\hline C & -3.407730 & 2.451020 & ๑. 859048 \\
\hline C & -4.790696 & 2.365522 & ๑. 873691 \\
\hline C & -5.440195 & 1.185632 & $\odot .482103$ \\
\hline C & 2.465778 & -1.026310 & ๑. 420945 \\
\hline $\mathrm{N}$ & 1.312800 & -1.208154 & -0.524870 \\
\hline $\mathrm{N}$ & -1.312795 & -1.208038 & $\odot .524853$ \\
\hline C & 2.589444 & 1.363085 & -0.450677 \\
\hline 0 & 1.265999 & 1.516171 & -0.458314 \\
\hline C & 1.875045 & -0.718661 & 1.829052 \\
\hline 0 & 2.481002 & -1.017756 & 2.863071 \\
\hline C & -2.465809 & -1.026343 & -0.420947 \\
\hline C & -1.875144 & -0.718886 & -1.829130 \\
\hline 0 & -2.481190 & -1.018064 & -2.863071 \\
\hline 0 & -0.692337 & -0.109849 & -1.754512 \\
\hline C & -2.589443 & 1.363146 & $\odot .450377$ \\
\hline 0 & -1.266001 & 1.516207 & $\odot .457953$ \\
\hline C & -0.560598 & -2.511840 & 0.531831 \\
\hline C & 0.560572 & -2.511939 & -0.531633 \\
\hline $\mathrm{Fe}$ & $\odot .000002$ & ๑. 197125 & -0.000166 \\
\hline 0 & ๑. 692284 & -0.109569 & 1.754273 \\
\hline $\mathrm{H}$ & 5.146707 & -0.821536 & ๑.233905 \\
\hline $\mathrm{H}$ & 6.521848 & 1.117014 & -0.491726 \\
\hline H & 5.375783 & 3.223048 & -1.192528 \\
\hline $\mathrm{H}$ & 2.895288 & 3.358071 & -1.155213 \\
\hline $\mathrm{H}$ & -5.146745 & -0.821542 & -0.233845 \\
\hline $\mathrm{H}$ & -2.895246 & 3.358217 & 1.154692 \\
\hline $\mathrm{H}$ & -5.375741 & 3.223209 & 1.192128 \\
\hline H & -6.521848 & 1.117102 & ๑. 491619 \\
\hline H & 3.099798 & -1.918004 & $\odot .476305$ \\
\hline $\mathrm{H}$ & 1.591849 & -0.960825 & -1.481468 \\
\hline $\mathrm{H}$ & -1.591776 & -0.960501 & 1.481416 \\
\hline H & -3.099825 & -1.918049 & -0.476157 \\
\hline H & -1.236757 & -3.358129 & $\odot .354909$ \\
\hline $\mathrm{H}$ & -0.123920 & -2.600778 & 1.527988 \\
\hline H & 1.236710 & -3.358217 & -0.354581 \\
\hline $\mathrm{H}$ & ๑. 123887 & -2.601018 & -1.527 \\
\hline
\end{tabular}


2b: $E=-1379.69983$

\begin{tabular}{|c|c|c|c|}
\hline C & -3.362753 & -0.103937 & -0.057487 \\
\hline C & -4.749132 & ๑. 064591 & -0.036436 \\
\hline C & -5.617602 & -0.958509 & -0.418108 \\
\hline C & -5.079546 & -2.181531 & -0.834104 \\
\hline C & -3.704137 & -2.371397 & -0.859686 \\
\hline C & 4.748838 & ๑. . 064529 & 0.034482 \\
\hline$C$ & 3.362454 & -0.103812 & ๑. .057113 \\
\hline$C$ & 3.704472 & -2.370920 & 0.860047 \\
\hline C & 5.079872 & -2.181229 & ๑. 832881 \\
\hline$C$ & 5.617613 & -0.958475 & $\odot .415700$ \\
\hline C & -2.462933 & 1.032320 & 0.403145 \\
\hline $\mathrm{N}$ & -1.324704 & 1.169334 & -0.553089 \\
\hline $\mathrm{N}$ & 1.324718 & 1.169547 & $\odot .553765$ \\
\hline C & -2.803599 & -1.353388 & -0.468040 \\
\hline 0 & -1.485290 & -1.584707 & -0.501783 \\
\hline$C$ & -1.895003 & ๑. 697416 & 1.825918 \\
\hline 0 & -2.492892 & 1.092463 & 2.836819 \\
\hline$C$ & 2.462409 & 1.032453 & -0.403093 \\
\hline$C$ & 1.893489 & ๑. 697486 & -1.825427 \\
\hline 0 & 2.489668 & 1.093903 & -2.836819 \\
\hline 0 & $\odot .789223$ & -0.027500 & -1.783460 \\
\hline$C$ & 2.803602 & -1.353013 & ๑. 468878 \\
\hline 0 & 1.485309 & -1.584239 & $\odot .504247$ \\
\hline C & ๑. 539175 & 2.434541 & 0.553916 \\
\hline$C$ & -0.539369 & 2.434474 & -0.553123 \\
\hline $\mathrm{Fe}$ & $-\odot .0 \odot \odot \odot 84$ & $-\odot .511792$ & $\odot .0 \odot \odot 979$ \\
\hline 0 & $-\odot .789670$ & -0.026029 & 1.784842 \\
\hline $\mathrm{H}$ & -5.151600 & 1.017740 & ๑. 294014 \\
\hline $\mathrm{H}$ & -6.690348 & -0.804918 & -0.393103 \\
\hline $\mathrm{H}$ & -5.738490 & -2.988845 & -1.138056 \\
\hline $\mathrm{H}$ & -3.272598 & -3.313608 & -1.174589 \\
\hline $\mathrm{H}$ & 5.151053 & 1.017479 & -0.296852 \\
\hline $\mathrm{H}$ & 3.273168 & -3.312925 & 1.175892 \\
\hline $\mathrm{H}$ & 5.739051 & -2.988471 & 1.136521 \\
\hline $\mathrm{H}$ & 6.690348 & $-\odot .805013$ & $\odot .389456$ \\
\hline $\mathrm{H}$ & -3.049991 & 1.955787 & ๑. 481660 \\
\hline $\mathrm{H}$ & -1.603483 & ๑. 916589 & -1.506242 \\
\hline $\mathrm{H}$ & 1.604157 & $\odot .917154$ & 1.506817 \\
\hline $\mathrm{H}$ & 3.049419 & 1.955910 & -0.482026 \\
\hline $\mathrm{H}$ & 1.187050 & 3.313608 & ○. 417978 \\
\hline $\mathrm{H}$ & ๑. . 057312 & 2.498867 & 1.531630 \\
\hline $\mathrm{H}$ & -1.187439 & 3.313380 & -0.417073 \\
\hline $\mathrm{H}$ & -0.057514 & 2.499148 & -1.530832 \\
\hline
\end{tabular}

3a: $E=-1379.67688$

$\begin{array}{lrrr}\text { C } & 3.211683 & -0.169478 & 0.088000 \\ \text { C } & 4.578046 & -0.122506 & -0.195596 \\ \text { C } & 5.195581 & 1.056305 & -0.616715 \\ \text { C } & 4.418789 & 2.213698 & -0.749093 \\ \text { C } & 3.057728 & 2.186746 & -0.471653 \\ \text { C } & 2.586144 & -1.425931 & 0.630822 \\ \text { N } & 1.286026 & -1.742263 & -0.045577 \\ \text { N } & -0.788046 & -0.172144 & -1.117129 \\ \text { C } & 2.411762 & 1.000366 & -0.046353\end{array}$




\begin{tabular}{|c|c|c|c|}
\hline C & 1.130098 & -1.819306 & -1.543846 \\
\hline 0 & 1.089852 & 1.009182 & 0.165836 \\
\hline $\mathrm{Fe}$ & -0.010372 & $-\odot .449455$ & ๑. 685481 \\
\hline 0 & 0.941495 & -0.777815 & 2.307278 \\
\hline C & 2.182319 & -1.217287 & 2.153229 \\
\hline 0 & 3.012018 & -1.440783 & 3.040627 \\
\hline 0 & -1.362503 & $\odot .616290$ & 1.438933 \\
\hline 0 & -2.675066 & -3.142475 & -0.539605 \\
\hline C & -2.143333 & $-\odot .812841$ & -1.056376 \\
\hline C & -2.587305 & 0.749368 & 0.924752 \\
\hline C & -3.518714 & 1.579459 & 1.605354 \\
\hline C & -4.809517 & 1.775180 & 1.141811 \\
\hline C & -5.248668 & 1.151947 & -0.035110 \\
\hline C & -4.358150 & $\odot .334109$ & -0.726967 \\
\hline C & -3.050478 & $\odot .12426 \odot$ & $-\odot .278971$ \\
\hline 0 & -1.085945 & -2.048762 & $\odot .699787$ \\
\hline C & -1.995590 & -2.146424 & -0.271084 \\
\hline C & $\odot .252268$ & -0.656114 & -2.099018 \\
\hline $\mathrm{H}$ & 5.167726 & -1.026479 & -0.070666 \\
\hline $\mathrm{H}$ & 6.258211 & 1.074875 & -0.829483 \\
\hline $\mathrm{H}$ & 4.881375 & 3.142475 & -1.068825 \\
\hline $\mathrm{H}$ & 2.446854 & 3.077056 & -0.561450 \\
\hline $\mathrm{H}$ & 3.294344 & -2.258655 & ๑.570826 \\
\hline $\mathrm{H}$ & $\odot .857182$ & -2.577333 & 0.378318 \\
\hline $\mathrm{H}$ & $-\odot .880101$ & 0.847035 & -1.197928 \\
\hline $\mathrm{H}$ & 0.661702 & -2.780607 & -1.772478 \\
\hline $\mathrm{H}$ & 2.112472 & -1.777349 & -2.023216 \\
\hline $\mathrm{H}$ & -2.556660 & -1.013546 & -2.050453 \\
\hline $\mathrm{H}$ & -3.169041 & 2.050042 & 2.516027 \\
\hline $\mathrm{H}$ & -5.485882 & 2.417359 & 1.697951 \\
\hline $\mathrm{H}$ & -6.258211 & 1.301100 & $-\odot .4 \odot \odot 256$ \\
\hline $\mathrm{H}$ & -4.680588 & $-\odot .164091$ & -1.637562 \\
\hline $\mathrm{H}$ & $\odot .893642$ & $\odot .206641$ & -2.281938 \\
\hline $\mathrm{H}$ & & $\odot$. & -3.04062 \\
\hline
\end{tabular}

3b: $E=-1379.69105$

$\begin{array}{lccr}\mathrm{C} & 3.335741 & -0.291982 & 0.161454 \\ \mathrm{C} & 4.633637 & -0.195742 & -0.343326 \\ \mathrm{C} & 5.218295 & 1.038234 & -0.635741 \\ \mathrm{C} & 4.477318 & 2.204151 & -0.412274 \\ \mathrm{C} & 3.184024 & 2.131623 & 0.090389 \\ \mathrm{C} & 2.761454 & -1.647375 & 0.513974 \\ \mathrm{~N} & 1.422320 & -1.869146 & -0.101363 \\ \mathrm{~N} & -0.601509 & -0.067115 & -0.862998 \\ \mathrm{C} & 2.572012 & 0.891321 & 0.399549 \\ \mathrm{C} & 1.179914 & -1.704317 & -1.562314 \\ \mathrm{O} & 1.325872 & 0.857062 & 0.874117 \\ \mathrm{Fe} & 0.093291 & -0.640463 & 1.145378 \\ \mathrm{O} & 1.275341 & -1.485127 & 2.439440 \\ \mathrm{C} & 2.525768 & -1.759896 & 2.075310 \\ \mathrm{O} & 3.462310 & -2.061548 & 2.819229 \\ \mathrm{O} & -1.449065 & 0.285184 & 1.744016 \\ 0 & -2.270918 & -3.173994 & -1.060307 \\ \mathrm{C} & -1.921692 & -0.761716 & -0.986698 \\ \mathrm{C} & -2.653124 & 0.430584 & 1.173086 \\ \mathrm{C} & -3.687340 & 1.077448 & 1.889257 \\ \mathrm{C} & -4.953383 & 1.257451 & 1.348221 \\ \mathrm{C} & -5.241922 & 0.800298 & 0.057698 \\ \mathrm{C} & -4.236108 & 0.167310 & -0.672216\end{array}$




$\begin{array}{rrrr}\text { C } & -2.954263 & -0.024003 & -0.148931 \\ \text { O } & -1.046551 & -2.279169 & 0.662085 \\ \text { C } & -1.758168 & -2.223656 & -0.449238 \\ \text { C } & 0.462135 & -0.365677 & -1.866845 \\ \text { H } & 5.198599 & -1.110126 & -0.502347 \\ \text { H } & 6.228517 & 1.089299 & -1.025282 \\ \text { H } & 4.914392 & 3.173994 & -0.629772 \\ \text { H } & 2.603540 & 3.027298 & 0.277488 \\ \text { H } & 3.485334 & -2.423173 & 0.234303 \\ \text { H } & 0.996668 & -2.741409 & 0.232777 \\ \text { H } & -0.728534 & 0.945715 & -0.767736 \\ \text { H } & 0.545736 & -2.536118 & -1.883632 \\ \text { H } & 2.119222 & -1.736532 & -2.126608 \\ \text { H } & -2.257969 & -0.817203 & -2.028850 \\ \text { H } & -3.449853 & 1.421022 & 2.888594 \\ \text { H } & -5.721236 & 1.754557 & 1.932641 \\ \text { H } & -6.228517 & 0.934958 & -0.370615 \\ \text { H } & -4.446698 & -0.196712 & -1.673741 \\ \text { H } & 1.186109 & 0.447585 & -1.793307 \\ \text { H } & 0.054644 & -0.397533 & -2.888594\end{array}$

4a: $E=-1379.65137$

$\begin{array}{lrrr}\text { C } & 3.138336 & -0.607571 & -0.534013 \\ \mathrm{C} & 4.478672 & -0.987124 & -0.459076 \\ \mathrm{C} & 5.510737 & -0.060486 & -0.610680 \\ \mathrm{C} & 5.183963 & 1.279378 & -0.847378 \\ \mathrm{C} & 3.855514 & 1.677223 & -0.931459 \\ \mathrm{C} & 2.039111 & -1.659456 & -0.408389 \\ \mathrm{~N} & 0.940137 & -0.945843 & 0.327014 \\ \mathrm{~N} & -0.971989 & 0.839473 & 0.412347 \\ \mathrm{C} & 2.788598 & 0.761183 & -0.782331 \\ \mathrm{O} & 1.520376 & 1.231869 & -0.836594 \\ \mathrm{C} & 1.601224 & -1.973341 & -1.887305 \\ \mathrm{O} & 1.966685 & -3.018533 & -2.435582 \\ \mathrm{O} & -1.385677 & -1.177345 & -1.264788 \\ \mathrm{O} & -2.424286 & 3.018533 & -2.012234 \\ \mathrm{C} & -2.241612 & 1.367950 & -0.223974 \\ \mathrm{C} & -2.696337 & -0.949430 & -1.099619 \\ \mathrm{C} & -3.633241 & -1.948166 & -1.456071 \\ \mathrm{C} & -4.995883 & -1.766676 & -1.256594 \\ \mathrm{C} & -5.482431 & -0.581900 & -0.688698 \\ \mathrm{C} & -4.574731 & 0.414887 & -0.329583 \\ \mathrm{C} & -3.201764 & 0.248906 & -0.525121 \\ \mathrm{O} & -0.849507 & 1.361418 & -2.217852 \\ \mathrm{C} & -1.815534 & 2.024204 & -1.601754 \\ \mathrm{C} & -0.187466 & -1.505074 & 1.126621 \\ \mathrm{C} & -1.070469 & -\Theta .247067 & 1.485529 \\ \mathrm{Fe} & 0.036619 & 0.081927 & -1.130574 \\ \mathrm{O} & 0.949634 & -0.955709 & -2.431833 \\ \mathrm{H} & 4.713300 & -2.033949 & -0.288104 \\ \mathrm{H} & 6.545793 & -0.376818 & -0.550791 \\ \mathrm{H} & 5.971955 & 2.015394 & -0.973578 \\ \mathrm{H} & 3.589231 & 2.708431 & -1.129280 \\ \mathrm{H} & 2.392946 & -2.562707 & 0.094721 \\ \mathrm{H} & 1.388289 & -0.212059 & 0.887602 \\ \mathrm{H} & -0.396628 & 1.627539 & 0.735641\end{array}$




$\begin{array}{rrrr}\text { H } & -2.720176 & 2.130203 & 0.397573 \\ \mathrm{H} & -3.243517 & -2.854378 & -1.903536 \\ \mathrm{H} & -5.688306 & -2.550388 & -1.548008 \\ \mathrm{H} & -6.545793 & -0.437266 & -0.536396 \\ \mathrm{H} & -4.933730 & 1.346907 & 0.097828 \\ \mathrm{H} & 0.154619 & -1.998775 & 2.045888 \\ \mathrm{H} & -0.744349 & -2.186508 & 0.490496 \\ \mathrm{H} & -2.116864 & -0.542505 & 1.575294 \\ \mathrm{H} & -0.733351 & 0.181863 & 2.435582\end{array}$

4b: $E=-1379.67326$

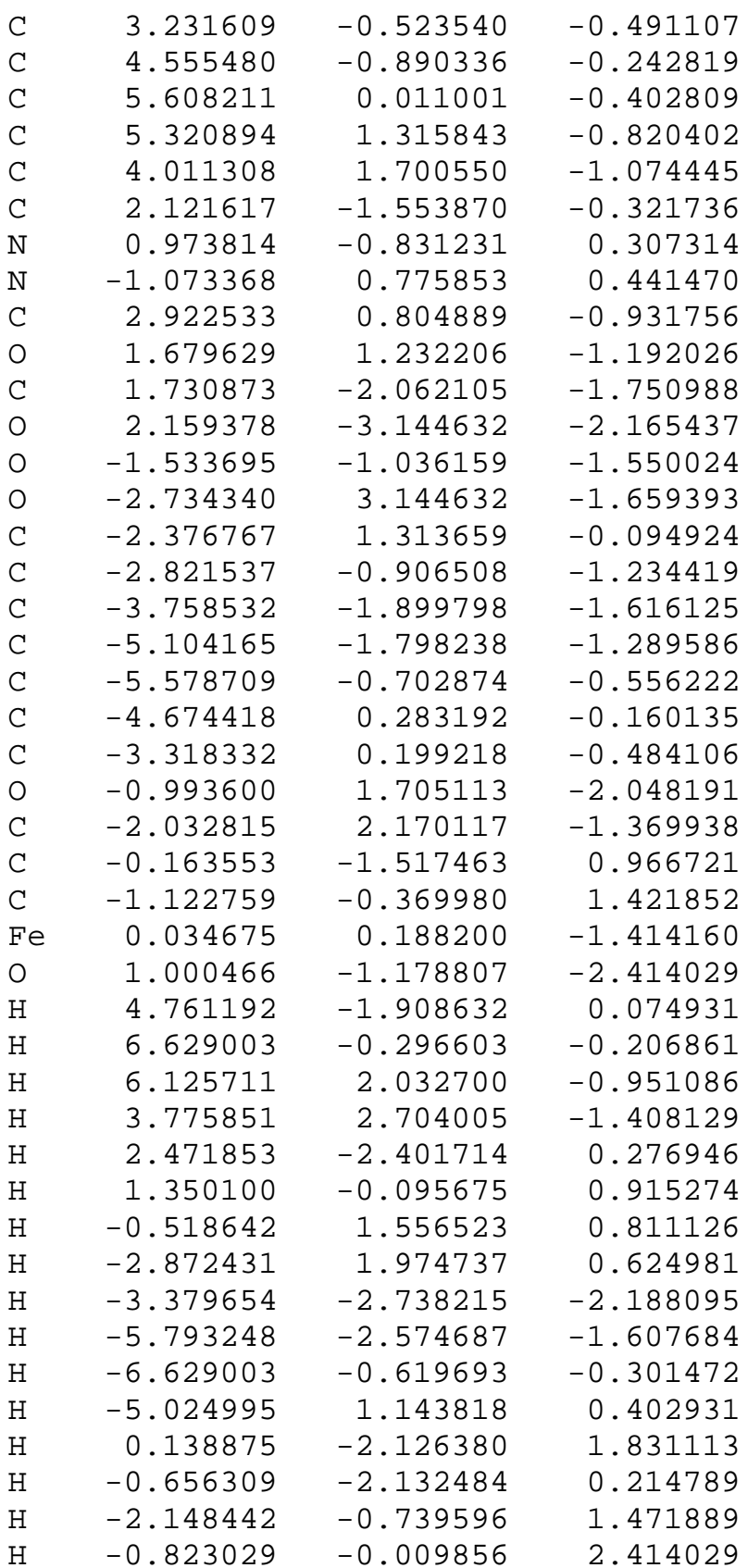


Plot of the Temperature dependence of $\chi \mathrm{T}$ for the meso $\mathrm{Na}[\mathrm{Fe}(\mathrm{III})$-EDDHA] isomer. The solid line represents the best fit of the data to equation (1) of the text:

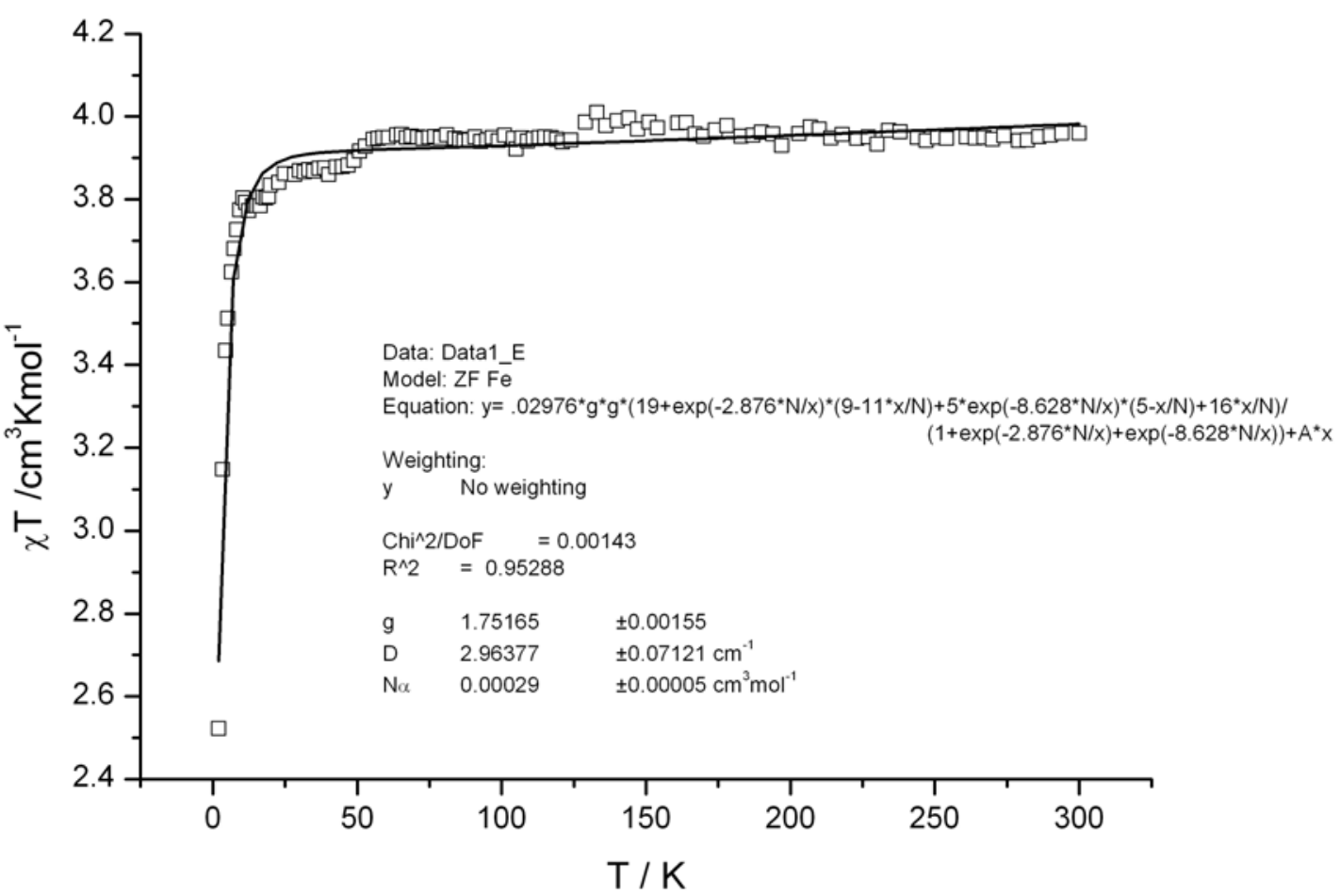

